Background and aims Ozanimod (RPC1063) is a specific and potent small molecule modulator of $\mathrm{S}_{1} \mathrm{P}_{1}, 5 \mathrm{R}$ that has shown therapeutic benefit in clinical trials of relapsing multiple sclerosis and ulcerative colitis. Its metabolite, RP-101075, shares ozanimod's specificity profile at the S1P receptor family in vitro, and its pharmacokinetic (PK) and pharmacodynamic profile in vivo.

Methods The $(\mathrm{NZB} \times \mathrm{NZW}) \mathrm{F} 1$ model was used in therapeutic dosing mode to assess the benefit of an $\mathrm{S}_{1} \mathrm{P}_{1,5 \mathrm{R}}$ modulator in systemic lupus erythematosus (SLE), compared to cyclophosphamide.

Results As predicted for an $\mathrm{S}_{1} \mathrm{P}_{1,5 \mathrm{R}}$ modulator, treatment with 0.3, 1 and $3 \mathrm{mg} / \mathrm{kg} \mathrm{RP}-101075$ resulted in a dose-dependent reduction in circulating $\mathrm{T}$ and $\mathrm{B}$ cells, achieving 62\%99\% decrease across all doses tested. Compared to vehicle treated animals, $3 \mathrm{mg} / \mathrm{kg} \mathrm{RP}-101075$ reduced proteinuria over the duration of the study (34 \pm 5 vs $18 \pm 1 \quad \mathrm{U}^{*}$ week; $\mathrm{p}<0.0001)$, and blood urea nitrogen $(36 \pm 5$ vs $21 \pm 3 \mathrm{mg} / \mathrm{dL}$; $\mathrm{p}<0.0001)$. Additionally, RP-101075 reduced kidney disease in a dose dependent manner, as quantified by histological assessment of mesangial expansion, endo- and exo-capillary proliferation, interstitial infiltrates and fibrosis, glomerular deposits and tubular atrophy. In addition, RP-101075 significantly reduced expression of fibrotic and immune genes in the kidneys, with minimal effect on IFN-inducible genes. Of particular note, RP-101075 lowered the number of plasmacytoid dendritic cells, a major source of IFN $\alpha$ in lupus patients, and all $\mathrm{B}$ and $\mathrm{T}$ cell subsets in the spleen.

Conclusions Given that RP-101075 shares the pharmacokinetic profile of ozanimod and reduces circulating lymphocytes similarly, ozanimod warrants clinical evaluation as a potential treatment for SLE.

\section{IS SPLENECTOMY A SAFE AND EFFECTIVE THERAPEUTIC OPTION IN SYSTEMIC LUPUS ERYTHEMATOSUS? A SINGLE-CENTRE STUDY}

A Barrera-Vargas*, S Morales-Padilla, R Vázquez-Rodríguez, R Reyna-de-la Garza, J Merayo-Chalico, J Alcocer-Varela, D Gómez-Martín. Instituto Nacional de Ciencias Médicas y Nutrición Salvador Zubirán, Immunology and Rheumatology, Mexico City, Mexico

\subsection{6/lupus-2017-000215.80}

Background and aims Thrombocytopenia is a relatively common feature in systemic lupus erythematosus (SLE) patients, although severe thrombocytopenia is rare. Splenectomy is considered an acceptable treatment option for refractory thrombocytopenia in different haematological conditions. However, its role in SLE has been controversial, due to potential surgical complications and to its possible association with SLE flares. The aim of this study was to determine safety and efficacy of splenectomy in a cohort of SLE patients.

Methods We included all patients with SLE who fulfilled $\geq 4$ ACR criteria, and underwent splenectomy between 2000 and 2015 in a tertiary care centre in Mexico City. Patients with other rheumatic diseases (except for anti-phospholipid syndrome) were excluded. We recorded demographic, clinical and serological characteristics at the time of surgery and during follow-up.

Results Thirty-six patients were included, 91.7\% were women and mean age was $33.31 \pm 12.95$. Refractory thrombocytopenia was the surgical indication in 28 patients $(77.7 \%)$. Laparoscopic splenectomy was performed in $80.6 \%$ of cases. Two patients had surgical complications (intra-abdominal sepsis and pancreatic fistula). There were no deaths directly associated with the procedure. Among patients with thrombocytopenia, $85.7 \%$ achieved complete remission, in a mean period of 1.65 \pm 3.13 months. Cumulative prednisone dose in the year after the surgery was lower than the year before $(8.7 \pm 5.8$ vs 4.2 \pm 3.2 grams, $\mathrm{p}<0.01)$. Mean SLEDAI score at baseline was $3.53 \pm 2.9$, and it decreased at 3 and 6 months $(1.15 \pm 2.07$ and $1.21 \pm 2.38, \mathrm{p}<0.01$, respectively) during follow-up.

Conclusions Splenectomy is a safe procedure in SLE patients, and it represents an effective therapeutic option for refractory thrombocytopenia.

\section{OUTCOMES OF MULTI-TARGET THERAPY USING MYCOPHENOLATE MOFETIL AND TACROLIMUS FOR REFRACTORY LUPUS NEPHRITIS}

CB Choi*, S Won, HH Kwon, SW Nam, SC Bae. Hanyang University Hospital for Rheumatic Diseases, Rheumatology, Seoul, Republic of Korea

\subsection{6/lupus-2017-000215.81}

Background and aims Outcomes of systemic lupus erythematosus (SLE) has significantly improved over the years. However, it can still be unfavourable when there is major organ involvement such as lupus nephritis and standard therapies fail. Outcomes of multi-target therapy using mycophenolate mofetil (MMF) and tacrolimus in SLE patients who were refractory to standard therapy was assessed.

Methods Retrospective analysis was done in patients with biopsy-confirmed lupus nephritis class III or IV who failed to achieve complete response with standard induction therapy for 24 weeks and switched to multi-target combination therapy with MMF and tacrolimus. Outcomes including renal response, urine protein/creatinine ratio (UPCR), glomerular filtration rate (GFR), serum albumin, and complements were assessed at 24 weeks.

Results A total of 20 patients, with mean age of $27.9 \pm 8.2$ years and $82.8 \%$ female, who initiated MMF and tacrolimus combination therapy were included. At 24 weeks, $25.9 \%$ showed complete response and $37.0 \%$ showed partial response. When all patients were compared, the mean UPCR increased from $3.06 \pm 3.00$ at baseline to $3.21 \pm 4.23$ at 24 weeks and GFR declined from $96.55 \pm 37.07 \mathrm{~mL} / \mathrm{min} / 1.73 \mathrm{~m}^{2}$ to $92.00 \pm 41.12 \mathrm{~mL} / \mathrm{min} / 1.73 \mathrm{~m}^{2}$. But in subgroup comparison, UPCR decreased from $1.82 \pm 0.86$ to $1.39 \pm 0.85$ in patients who had shown partial response to standard induction therapy and increased from $5.61 \pm 2.93$ to $8.62 \pm 4.11$ in no response patients.

Conclusions Multi-target therapy combining MMF and tacrolimus can be considered in patients who had partial response to standard induction therapy in patients with lupus nephritis.

\section{ETHANOL PREVENTS DAMAGE OF SKIN, KIDNEY AND JOINT IN LUPUS-PRONE MICE BY REGULATING LIPID RAFTS}

${ }^{1} \mathrm{H}$ Dou, ${ }^{2} \mathrm{~L}$ Liu, ${ }^{1,2} \mathrm{GM}$ Deng*. ${ }^{1}$ Nanjing Medical University, Key Laboratory of Antibody Techniques of Ministry of Health, Nanjing, China; ${ }^{2}$ Harvard Medical School, Beth Israel Deaconess Medical Centre, Boston, USA

\subsection{6/lupus-2017-000215.82}

Background and aims Ethanol has been elaborated to have a beneficial effect on destructive arthritis. Nevertheless, the 